\title{
Stress, Anxiety, Depression among Nurses Caring for COVID-19 Patients in Babol, Iran: A logestic Regression
}

\section{Parvin Aziznejadroshan}

Babol University of Medical Science Faculty of Nursing and Midwifery

Mojtaba Qanbari Qalehsari ( $\sim$ m2ghanbari@yahoo.com )

Babol University of Medical Sciences

Fatemeh Shaker Zavardehi

Islamic Azad University Babol Branch

\section{Research}

Keywords: Stress, anxiety, depression, COVID-19, nursing care, nurses

Posted Date: October 30th, 2020

DOI: https://doi.org/10.21203/rs.3.rs-98099/v1

License: (c) (i) This work is licensed under a Creative Commons Attribution 4.0 International License.

Read Full License 


\section{Abstract}

Introduction: Nurses who are involved in the caring of COVID-19 patients, are at risk of mental distress. The present study was conducted with the purpose of demonstrating the relationship between stress, anxiety, depression and characteristics of nurses who provide care to COVID-19 patients.

Methods: This descriptive cross-sectional study was conducted using the census method from May to June, 2020 on 224 clinical nurses who were working in hospitals affiliated with Babol University of Medical Sciences and were involved in caring for COVID-19 patients. The data collection instruments included the Depression, Anxiety and Stress Scale - 21 items (DASS-21) and the individual characteristic questionnaire. Bivariate and multivariate binary logistic regressions were computed to identify the associated factors.

Result: The mean and the standard deviation for stress, anxiety and depression scores were $9.47 \pm 7.30$, $9.29 \pm 7.51$ and $8.84 \pm 7.22$ respectively. $17.4 \%$ had stress, $54 \%$ had anxiety and $43 \%$ had a degree of depression. There were significant relationships between the nurses' stress level and characteristics including age $(\mathrm{OR}=3.009,95 \% \mathrm{Cl} 1.46-6.16, \mathrm{P}=0.003)$, having children $(\mathrm{OR}=0.26,95 \% \mathrm{Cl} 0.11-0.63$, $\mathrm{P}=0.003)$, work experience $(\mathrm{OR}=4.50,95 \% \mathrm{Cl} 2.17-9.96, \mathrm{P}=0.000)$ and employment status $(\mathrm{OR}=0.39,95 \% \mathrm{Cl}$ $0.16-0.95, P=0.04)$. Moreover, along with these characteristics, job satisfaction ( $\mathrm{OR}=3.03,95 \% \mathrm{Cl} 5.64-$ $1.63, \mathrm{P}=0.000)$, level of physical activities $(\mathrm{OR}=0.26,95 \% \mathrm{Cl} 0.08-0.82, \mathrm{P}=0.02)$, exercising $(\mathrm{OR}=2.27$, $95 \% \mathrm{Cl} 1.31-3.90, \mathrm{P}=0.003)$ and violence in the workplace $(\mathrm{OR}=0.27,95 \% \mathrm{Cl} 0.12-0.56, \mathrm{P}=0.001)$ also had significant relationships with the nurses' anxiety level. Furthermore, the relationships between the nurses' level of depression and characteristics including age $(\mathrm{OR}=2.07,95 \% \mathrm{Cl} 1.15-3.72, \mathrm{P}=0.014)$, work experience $(\mathrm{OR}=21.79,95 \% \mathrm{Cl} 1.04-3.10, \mathrm{P}=0.03)$, job satisfaction $(\mathrm{OR}=3.03,95 \% \mathrm{Cl} 1.63-5.64$, $\mathrm{P}=0.000)$, exercising $(\mathrm{OR}=1.76,95 \% \mathrm{Cl} 1.02-3.04, \mathrm{P}=0.04)$, having chronic diseases $(\mathrm{OR}=0.35,95 \% \mathrm{Cl}$ $0.15-0.81, P=0.014)$, violence in the workplace $(\mathrm{OR}=0.39,95 \% \mathrm{Cl} 0.20-0.75, \mathrm{P}=0.005)$ and sleep (OR $=1.77,95 \% \mathrm{Cl} 1.00-3.16, \mathrm{P}=0.050$ ) were significant.

Conclusion: The authorities should consider a number of the individual characteristics of nurses including age, work experience, gender, marital status, having children, job satisfaction, sleep, violence in the workplace and history of chronic disease in their planning and provide psychological support for them.

\section{Background}

The COVID-19 pandemic is a serious threat for public health (1). Nurses work at the frontline of the fight against this disease and risk their lives while performing their duties (2). They have always played an important role in infection prevention and control, isolation and public health (3). In the early days of the outbreak of COVID-19, more than 3000 medical staff in Wuhan, China, got infected (40\% in hospitals and $60 \%$ in the society) (3). 
In the face of this critical situation, the healthcare workers at the forefront, who are directly involved in the diagnosis, treatment and caring of COVID-19 patients, are at risk of mental distress and other mental health disorders. The growing number of confirmed and suspected cases, the high level of work pressure, the lack of personal protective equipment, the extensive media coverage, the shortage of specific drugs and feeling that they are not being sufficiently supported may all affect the healthcare workers' stress (4). A whole new working condition, the job burn-out caused by the burden of heavy workload and the protective equipment, fear of getting infected and infecting others, a feeling of inability to attend to patients and managing the relationships in stressful situations are the challenges faced by the health care providers (5).

The emergency situations caused by COVID-19 put major pressures on nursing services. When nurses work in environments where job demands are high and resources are low, they are exposed to higher job stress and more physical and psychological symptoms, which might have negative effects on their health and well-being $(6,7)$. Maintaining the mental health of the nursing staff is necessary to control infectious diseases (8).

The previous studies have shown that when nurses are in close contact with patients with emerging infectious diseases such as MERS, Covid-19 and H1N1, they suffer from loneliness, anxiety, fear, fatigue, sleep disorders and other mental and physical health problems $(9,10,11)$. A review study showed that demographic variables including gender, occupation, age, workplace and department as well as psychological variables such as self-efficacy and poor social support were accompanied by increase in stress, anxiety, depression symptoms and insomnia in healthcare workers. Evidences indicated that COVID-19 can be an independent risk factor for stress in healthcare workers (12). In a research in 2020 in China on mental health of healthcare workers who were exposed to COVID-19, the findings showed that $50.4 \%$ of them reported depression symptoms, $44.6 \%$ anxiety, $34 \%$ insomnia and $71.5 \%$ symptoms of distress (4). The findings of another study titled "Work Stress among Chinese Nurses to Support Wuhan for Fighting against COVID-19" showed that the mean self-reported stress and anxiety scores were 32.19 \pm 7.56 and $39.91 \pm 12.92$ respectively and the multiple regression analysis showed that having a child in the family, working hours per week and anxiety were the main factors influencing the nurses' stress (3).

Since COVID-19 is a new disease and the healthcare system and culture vary among different countries, more research is needed on the psychological experience of frontline nurses coping with COVID-19 (13). Regular screening of healthcare workers involved in patient care as well as diagnosing and providing care for patients with COVID-19 to assess their stress, depression and anxiety seems necessary (12). The existing information about work stress among nurses is limited (3).

Considering the important role of nurses in improving and promoting healthcare services in the society, the researchers decided to investigate the relationship between stress, anxiety, depression and some of the individual characteristics of nurses involved in caring for COVID-19 patients in hospitals affiliated with Babol University of Medical Sciences. 


\section{Methods}

\section{Study setting, design, population, and sampling technique}

This descriptive cross-sectional study was conducted from May to June, 2020 using census method on all clinical nurses working in two hospitals affiliated with Babol University of Medical Sciences (Ayatollah Rouhani and Shahid Yahya Nejad) in northern Iran who were involved in direct care of COVID-19 patients. Among the 302 nurses who directly involved in the care of Covid-19 patients, 224 completed the questionnaire. The inclusion criteria were: having a bachelor's or master's degree in nursing, being directly involved in providing care for COVID-19 patients in one of the wards of the two aforementioned hospitals, at least one year experience of working in the hospital and willingness to participate in the study.

\section{Data collection procedure and tools}

The research instruments used in this study included the individual characteristics questionnaire and the short form version of Depression Anxiety Stress Scales (DASS-21). The individual characteristics questionnaire had 18 items comprising age, gender, marital status, level of education, work experience, type of work shift, level of job satisfaction, average amount of overtime, employment status, type of hospital, having children, level of physical activity, exercising, history of chronic diseases, workplace violence, sleep and history of depression in the family. DASS-21 is a self-report instrument that shows the recent mood swings and was first introduced by Lovibond in 1995 (14). As the name suggests, it has 21 items which are scored on Likert scales. These 21 items contain: 7 questions related to stress including finding it hard to wind down, tendency to over-react to situations, using a lot of nervous energy, getting agitated, finding it difficult to relax, being intolerant of anything that keeps you from getting on with what you were doing, and feeling touchy; 7 questions related to anxiety including dryness of the mouth, breathing difficulty, trembling, being worried about situations in which you might panic and make a fool of yourself, feeling close to panic, action of the heart in the absence of physical exertion, and feeling scared without any good reason; and finally 7 questions related to depression including inability to experience any positive feeling at all, finding it difficult to work up the initiative to do things, feeling that you have nothing to look forward to, feeling down-hearted and blue, being unable to become enthusiastic about anything, feeling that you are not worth much as a person, and feeling that life is meaningless. The validity and reliability of this instrument have been reviewed and approved by Mehdipour and Najafi (15, 16). The internal consistency of the scale was determined in the study of Shamsaie et al. (2018) using Cronbach's alpha and the figures for each subscale were as follows: $85 \%$ for stress, $86 \%$ for anxiety and $83 \%$ for depression (17).

After defining the purpose of the study and reassuring the participants that all information will remain confidential, the researcher distributed the questionnaires and gave explanation in case any of the items were unclear. The participants had to read each statement and rate the intensity of the symptom mentioned in it on a 4-point Likert scale as it had been experienced over the past week. In this regard, zero meant that the symptom had not been experienced at all, one meant it had been experienced to some extent, two meant it had been experienced rather much, and three meant it had been experienced very 
much. The total score of each subscale of this instrument is obtained by summing up the scores of the questions related to that subscale.

Since DASS-21 is the short-form version of the main scale (42-item), the total score of each subscale must be doubled (16). Then, the intensity of the signs and symptoms can be determined according to Table1.

Table 1

Symptom severity of different parameters in the three groups

\begin{tabular}{|llll|}
\hline & stress & anxiety & depression \\
\hline normal & $0-14$ & $0-7$ & $0-9$ \\
\hline mild & $15-18$ & $8-9$ & $10-13$ \\
\hline moderate & $19-25$ & $10-14$ & $14-20$ \\
\hline severe & $26-33$ & $15-19$ & $21-27$ \\
\hline Extremely severe & $>34$ & $>20$ & $>28$ \\
\hline $\begin{array}{l}\text { Lovibond, S.H. \& Lovibond, P.F. (1995). Manual for the Depression Anxiety \& Stress Scales. (2nd } \\
\text { Ed.)Sydney: Psychology Foundation. }\end{array}$ & \\
\hline
\end{tabular}

\section{Data processing and analysis}

Data analysis was performed using SPSS statistical software version 18. The correlations between independent and dependent variables were tested by binary logistic regression analysis. The variables which had a p-value less than 0.2 were entered into multivariate analysis. In the end, the model was finalized using backward elimination. Finally, a p-value of less than 0.05 was considered statistically significant association, and the adjusted odds ratio with $95 \% \mathrm{Cl}$ was calculated to determine the strength of association.

\section{Data quality management}

Regular monitoring of data collection was performed by the principal investigator to ensure that all necessary data were properly collected. In every stage of data collection, the filled questioners were cheeked manually first for completeness and consistency then the collected data were processed timely and enter from a paper onto the computer twice. If a questionnaire is incomplete, it will be returned for completion, if the respondents were not willing to fill the missed information, the questionnaire was discarded from the analysis for gross incompleteness.

\section{Ethical approval and consent to participate}

The study protocol was approved by the Research Deputy of Babol University of Medical Sciences. Written informed consent was obtained from each study participant. The involvement of the study 
participants was voluntarily and participants were informed of the right to withdraw anytime from the study.

\section{Results}

\section{Demographic Characteristics:}

The study sample contained 224 nurses with an average age of $32.9 \pm 6.31$. The demographic characteristics of the participants were as follows: 167 (74.6\%) were female, $148(68 \%)$ were younger than 50 years of age, $85 \%$ had bachelor's degree, $80 \%$ worked rotating shifts, $58 \%$ had 1 to 10 years of work experience, $67 \%$ were working under a formal contract, $80 \%$ were married, $59 \%$ had at least one child, $52 \%$ were working 50 to 100 hours of overtime per month, $67 \%$ said they were satisfied with their jobs, $90 \%$ had physical activities, $53 \%$ did not exercise regularly, $87 \%$ did not have any chronic diseases, $98 \%$ did not have any history of mental disorders, $90 \%$ had no history of depression in their families, $68 \%$ got 7 to 8 hours of sleep at least 3 to 4 days a week, and $78 \%$ did not experience workplace violence (Table 2). 
Table 2

Frequency distribution of respondents by socio-demographic characteristics and selected variables

\begin{tabular}{|c|c|c|c|c|}
\hline variables & mean & Standard deviation & number & percentage \\
\hline \multicolumn{5}{|l|}{ sex } \\
\hline male & & & 57 & 25.4 \\
\hline female & & & 167 & 76.4 \\
\hline age & 32.9 & 6.31 & & \\
\hline$\leq 35$ & & & 148 & 68 \\
\hline$>35$ & & & 68 & 32 \\
\hline \multicolumn{5}{|l|}{ Shift type } \\
\hline morning & & & 23 & 10.3 \\
\hline Evening or night & & & 22 & 9.8 \\
\hline rotational & & & 178 & 79.8 \\
\hline \multicolumn{5}{|l|}{ Work Experience } \\
\hline$\leq 10$ & & & 129 & 57.6 \\
\hline$>10$ & & & 95 & 42.5 \\
\hline \multicolumn{5}{|l|}{ Education level } \\
\hline bachelor of Nursing & & & 191 & 85.3 \\
\hline Master of Nursing & & & 33 & 14.7 \\
\hline \multicolumn{5}{|l|}{ Marital status } \\
\hline single & & & 43 & 19.2 \\
\hline married & & & 181 & 80.8 \\
\hline \multicolumn{5}{|l|}{ Having a child } \\
\hline yes & & & 132 & 59.5 \\
\hline no & & & 90 & 40.5 \\
\hline \multicolumn{5}{|l|}{ Employment status } \\
\hline yes & & & 149 & 67 \\
\hline no & & & 71 & 33 \\
\hline \multicolumn{5}{|l|}{ Overtime(h) } \\
\hline$<50$ & & & 38 & 17 \\
\hline
\end{tabular}




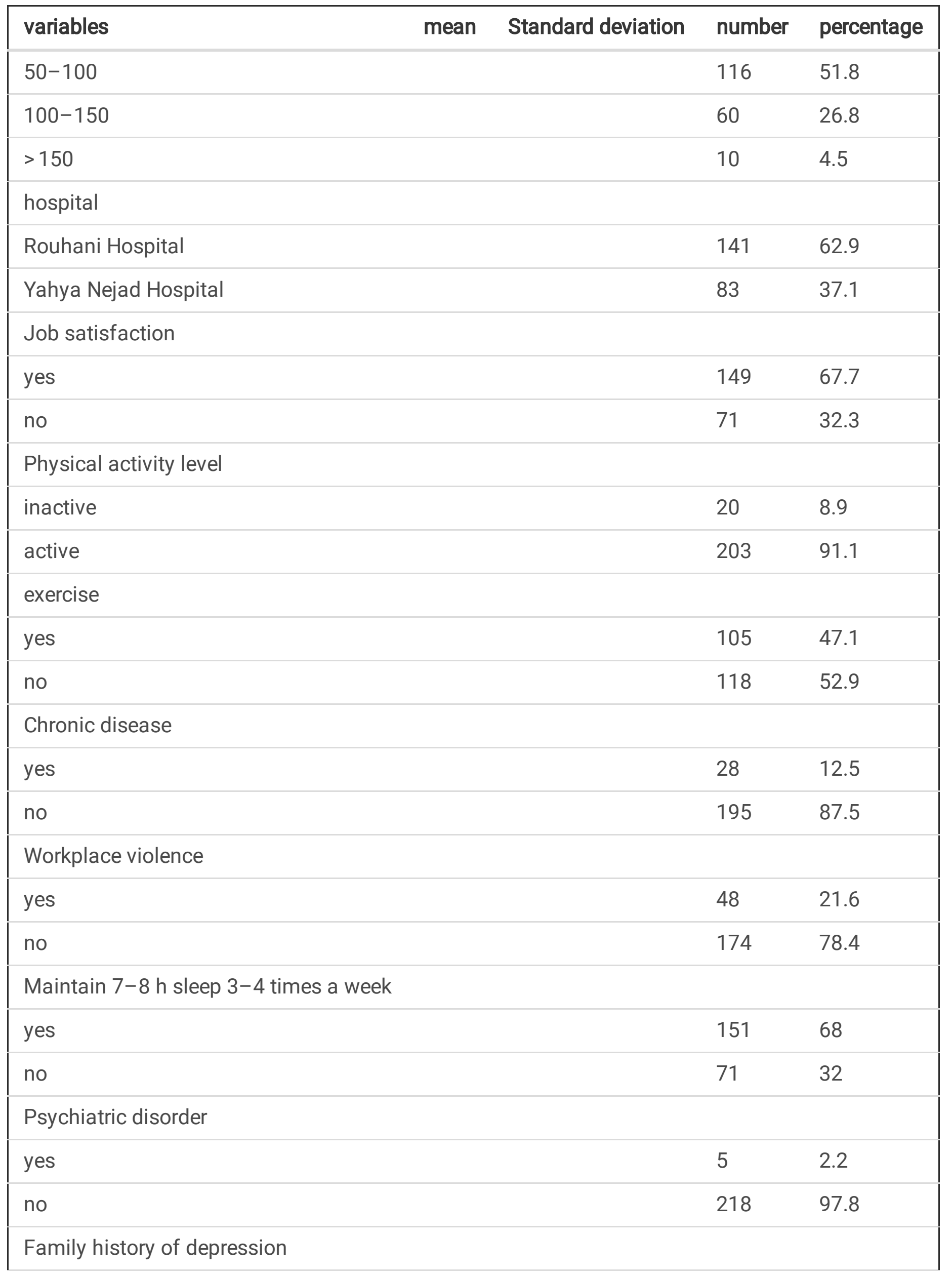




\begin{tabular}{|c|c|c|c|c|}
\hline variables & mean & Standard deviation & number & percentage \\
\hline yes & & & 22 & 9.9 \\
\hline no & & & 200 & 90.1 \\
\hline
\end{tabular}

\section{Level of Stress, Anxiety and Depression}

The mean ( \pm standard deviation) stress score calculated by DASS-21 was $4.73( \pm 3.65)$, which was multiplied by two (to make it equal to the result of the main scale) and the result was $9.47( \pm 7.30)$. More than $82 \%$ of the nurses had normal stress levels.

The mean ( \pm standard deviation) anxiety score was $4.67( \pm 3.75)$ based on DASS-21 and $9.34( \pm 7.51)$ after multiplying it by two. More than half of the nurses (54\%) had degrees of anxiety.

The mean ( \pm standard deviation) depression score was $4.42( \pm 3.61)$ as measured by DASS-21 and 8.84 $( \pm 7.22)$ after it was doubled. About $43 \%$ of the nurses experienced some degrees of depression (Table 3 ).

Table 3

Frequency distribution of nurses' depression, anxiety and stress

\begin{tabular}{|llll|}
\hline & stress & anxiety & depression \\
\cline { 2 - 4 } & Number (\%) & Number (\%) & Number (\%) \\
\hline normal & $181(82)$ & $100(45.7)$ & $127(57.7)$ \\
\hline mild & $15(6.7)$ & $26(11.9)$ & $42(19.1)$ \\
\hline moderate & $17(7.6)$ & $44(20.1)$ & $36(16.4)$ \\
\hline severe & $4(1.8)$ & $27(12.3)$ & $12(5.5)$ \\
\hline Very severe & $3(1.3)$ & $22(10)$ & $3(1.4)$ \\
\hline
\end{tabular}

\section{Factors Associated with Stress}

According to the results of the binary logistic regression variables including age, work experience, having children and employment status had significant relationships with the nurses' stress levels. In this regard, the nurses who were older than 35 years had three times greater risk of experiencing stress than the nurses who were younger than 35 years; the nurses who had more than 10 years of experience were 4.65 times as likely to feel stressed as the nurses who had less than 10 years of experience; the nurses who did not have children were 0.26 times less likely to get stressed than the nurses who had children; and the nurses who were employed in non-governmental hospitals were 0.39 times less likely to feel stressed than those employed in governmental hospitals (Table 4). 
Table 4

Frequency distribution of respondents by stress status and socio-demographic characteristics and other selected variables

\begin{tabular}{|c|c|c|c|c|c|c|c|}
\hline \multirow[t]{3}{*}{ variables } & \multicolumn{4}{|c|}{ stress status } & \multirow[t]{3}{*}{$\mathbf{P}$} & \multirow[t]{3}{*}{ OR } & \multirow[t]{3}{*}{$95 \% \mathrm{Cl}$} \\
\hline & yes & $\%$ & no & $\%$ & & & \\
\hline & (n) & & (n) & & & & \\
\hline \multicolumn{8}{|l|}{ sex } \\
\hline male & 9 & 16.07 & 47 & 83.93 & - & - & - \\
\hline female & 30 & 18.29 & 134 & 81.71 & 0.70 & 1.16 & $\begin{array}{l}2.64- \\
0.51\end{array}$ \\
\hline \multicolumn{8}{|l|}{ age } \\
\hline$\leq 35$ & 18 & 12.16 & 130 & 87.84 & - & - & - \\
\hline$>35$ & 20 & 29.41 & 48 & 70.59 & 0.003 & 3.009 & $\begin{array}{l}6.16- \\
1.46\end{array}$ \\
\hline \multicolumn{8}{|l|}{ Shift type } \\
\hline morning & 6 & 26.09 & 17 & 73.91 & - & - & - \\
\hline Evening or night & 3 & 15 & 17 & 85 & 0.37 & 0.49 & $\begin{array}{l}2.33- \\
0.10\end{array}$ \\
\hline rotational & 30 & 17.05 & 146 & 82.95 & 0.29 & 0.58 & $1.5-0.21$ \\
\hline \multicolumn{8}{|l|}{ Work Experience } \\
\hline$\leq 10$ & 11 & 8.59 & 117 & 91.41 & - & - & - \\
\hline$>10$ & 28 & 30.43 & 64 & 69.57 & 0.000 & 4.65 & $\begin{array}{l}9.96- \\
2.17\end{array}$ \\
\hline \multicolumn{8}{|l|}{ Education level } \\
\hline bachelor of Nursing & 35 & 18.62 & 153 & 81.38 & - & - & - \\
\hline Master of Nursing & 4 & 12.5 & 28 & 87.5 & 0.40 & 0.62 & $\begin{array}{l}1.89- \\
0.20\end{array}$ \\
\hline \multicolumn{8}{|l|}{ Marital status } \\
\hline single & 7 & 16.28 & 36 & 83.72 & - & - & - \\
\hline married & 32 & 18.08 & 145 & 81.92 & 0.78 & 1.13 & $\begin{array}{l}2.77- \\
0.46\end{array}$ \\
\hline \multicolumn{8}{|l|}{ Having a child } \\
\hline yes & 32 & 24.62 & 98 & 75.38 & - & - & - \\
\hline
\end{tabular}




\begin{tabular}{|c|c|c|c|c|c|c|c|}
\hline \multirow{2}{*}{$\begin{array}{l}\text { variables } \\
\text { no }\end{array}$} & \multicolumn{4}{|c|}{ stress status } & \multirow{2}{*}{$\begin{array}{l}\mathbf{P} \\
0.003\end{array}$} & \multirow{2}{*}{$\begin{array}{l}\mathrm{OR} \\
0.26\end{array}$} & \multirow{2}{*}{$\begin{array}{l}95 \% \mathrm{Cl} \\
0.63- \\
0.11\end{array}$} \\
\hline & 7 & 8.05 & 80 & 91.95 & & & \\
\hline \multicolumn{8}{|l|}{ Employment status } \\
\hline yes & 32 & 21.48 & 117 & 78.52 & - & - & - \\
\hline no & 7 & 9.86 & 64 & 90.14 & 0.04 & 0.39 & $\begin{array}{l}0.95- \\
0.16\end{array}$ \\
\hline \multicolumn{8}{|l|}{ Overtime(h) } \\
\hline$<50$ & 9 & 23.68 & 29 & 76.32 & - & - & - \\
\hline $50-100$ & 18 & 15.79 & 96 & 84.21 & 0.27 & 0.60 & $\begin{array}{l}1.48- \\
0.24\end{array}$ \\
\hline $100-150$ & 10 & 17.24 & 48 & 82.76 & 0.44 & 0.67 & $\begin{array}{l}1.84- \\
0.24\end{array}$ \\
\hline$>150$ & 2 & 20 & 8 & 80 & 0.80 & 0.80 & $4.5-0.14$ \\
\hline \multicolumn{8}{|l|}{ hospital } \\
\hline Rouhani Hospital & 28 & 20.44 & 109 & 79.56 & - & - & - \\
\hline Yahya Nejad Hospital & 11 & 13.25 & 72 & 86.75 & 0.17 & 0.59 & $\begin{array}{l}1.26- \\
0.27\end{array}$ \\
\hline \multicolumn{8}{|l|}{ Job satisfaction } \\
\hline yes & 21 & 14.48 & 124 & 85.52 & - & - & - \\
\hline no & 18 & 25.35 & 53 & 74.65 & 0.054 & 2.05 & $\begin{array}{l}4.06- \\
0.98\end{array}$ \\
\hline \multicolumn{8}{|l|}{ Physical activity level } \\
\hline inactive & 3 & 15.79 & 16 & 84.21 & - & - & - \\
\hline active & 36 & 18.09 & 163 & 81.91 & 0.80 & 1.17 & $\begin{array}{l}4.18- \\
0.32\end{array}$ \\
\hline \multicolumn{8}{|l|}{ exercise } \\
\hline yes & 15 & 14.42 & 89 & 85.58 & - & - & - \\
\hline no & 24 & 20.87 & 91 & 79.13 & 0.21 & 1.56 & $\begin{array}{l}3.17- \\
0.77\end{array}$ \\
\hline \multicolumn{8}{|l|}{ Chronic disease } \\
\hline yes & 4 & 14.29 & 24 & 85.71 & - & - & - \\
\hline
\end{tabular}




\begin{tabular}{|c|c|c|c|c|c|c|c|}
\hline \multirow{2}{*}{$\begin{array}{l}\text { variables } \\
\text { no }\end{array}$} & \multicolumn{4}{|c|}{ stress status } & \multirow{2}{*}{$\begin{array}{l}P \\
0.60\end{array}$} & \multirow{2}{*}{$\begin{array}{l}\text { OR } \\
1.34\end{array}$} & \multirow{2}{*}{$\begin{array}{l}95 \% \mathrm{Cl} \\
4.12- \\
0.43\end{array}$} \\
\hline & 35 & 18.32 & 156 & 81.68 & & & \\
\hline \multicolumn{8}{|c|}{ Workplace violence } \\
\hline yes & 9 & 19.15 & 38 & 80.85 & - & - & - \\
\hline no & 30 & 17.54 & 141 & 82.46 & 0.79 & 0.89 & $\begin{array}{l}2.05- \\
0.39\end{array}$ \\
\hline \multicolumn{8}{|c|}{$\begin{array}{l}\text { Maintain 7-8 h sleep 3-4 times a } \\
\text { week }\end{array}$} \\
\hline yes & 25 & 16.56 & 126 & 83.44 & - & - & - \\
\hline no & 14 & 20.9 & 153 & 79.10 & 0.44 & 1.33 & $\begin{array}{l}2.75- \\
0.64\end{array}$ \\
\hline \multicolumn{8}{|c|}{ Psychiatric disorder } \\
\hline yes & 1 & 25 & 3 & 75 & - & - & - \\
\hline no & 38 & 17.68 & 177 & 82.33 & 0.70 & 0.64 & $\begin{array}{l}6.36- \\
0.06\end{array}$ \\
\hline \multicolumn{8}{|c|}{ Family history of depression } \\
\hline yes & 7 & 31.82 & 15 & 68.18 & - & - & - \\
\hline no & 32 & 16.33 & 164 & 83.67 & 0.07 & 0.41 & $1.1-0.15$ \\
\hline
\end{tabular}

\section{Factors Associated with Anxiety}

According to the results of the binary logistic regression, there were significant relationships between the nurses' anxiety levels and the variables including age, work experience, having children, employment status, job satisfaction, level of physical activities, exercising and violence in the workplace. In this regard, the nurses who were older than 35 years had 2.61 times greater risk of suffering from an anxiety disorder than the nurses who were younger than 35 years; the nurses who had more than 10 years of experience were 3.25 times as likely to get anxious as those nurses who had less than 10 years of experience; the nurses who did not have children were less likely to feel anxious $(O R=0.45)$; the nurses who were employed in non-governmental hospitals or had an unclear employment status were less likely to experience anxiety than the nurses with a clear employment status $(\mathrm{OR}=0.46)$; the nurses who were not satisfied with their jobs felt anxious 3.3 times more than the nurses who were satisfied with their jobs; and the nurses who were active in terms of physical activities were far less likely to get anxious than the nurses who were physically inactive $(O R=0.26)$. Similar to physical activity, the nurses who did not exercise experienced anxiety 2.27 times more than the nurses that exercised regularly. Moreover, the nurses who did not experience workplace violence were less likely to get anxious $(\mathrm{OR}=0.27)$ (Table 5$)$. 
Table 5

Frequency distribution of respondents by anxiety status and socio-demographic characteristics and other selected variables

\begin{tabular}{|c|c|c|c|c|c|c|c|}
\hline \multirow[t]{3}{*}{ variables } & \multicolumn{4}{|c|}{ anxiety status } & \multirow[t]{3}{*}{$\mathbf{P}$} & \multirow[t]{3}{*}{ OR } & \multirow[t]{3}{*}{$95 \% \mathrm{Cl}$} \\
\hline & yes & $\%$ & no & $\%$ & & & \\
\hline & (n) & & (n) & & & & \\
\hline \multicolumn{8}{|l|}{ sex } \\
\hline male & 29 & 51.79 & 27 & 48.21 & - & - & - \\
\hline female & 90 & 55.21 & 73 & 44.79 & 0.65 & 1.14 & $\begin{array}{l}2.10- \\
0.62\end{array}$ \\
\hline \multicolumn{8}{|l|}{ age } \\
\hline$\leq 35$ & 70 & 47.30 & 78 & 52.70 & - & - & - \\
\hline$>35$ & 47 & 70.15 & 20 & 29.85 & 0.002 & 2.61 & $\begin{array}{l}4.84- \\
1.41\end{array}$ \\
\hline \multicolumn{8}{|l|}{ Shift type } \\
\hline morning & 13 & 56.52 & 10 & 43.48 & - & - & - \\
\hline Evening or night & 8 & 36.36 & 14 & 63.64 & 0.17 & 0.43 & $\begin{array}{l}1.45- \\
0.13\end{array}$ \\
\hline rotational & 97 & 56.07 & 76 & 43.93 & 0.96 & 0.98 & $\begin{array}{l}2.36- \\
0.40\end{array}$ \\
\hline \multicolumn{8}{|l|}{ Work Experience } \\
\hline$\leq 10$ & 54 & 42.52 & 73 & 57.48 & - & - & - \\
\hline$>10$ & 65 & 70.65 & 27 & 29.35 & 0.000 & 3.25 & $\begin{array}{l}5.75- \\
1.84\end{array}$ \\
\hline \multicolumn{8}{|l|}{ Education level } \\
\hline bachelor of Nursing & 103 & 55.38 & 83 & 44.62 & - & - & - \\
\hline Master of Nursing & 16 & 48.48 & 17 & 51.52 & 0.46 & 0.75 & $\begin{array}{l}1.59- \\
0.36\end{array}$ \\
\hline \multicolumn{8}{|l|}{ Marital status } \\
\hline single & 23 & 53.49 & 20 & 46.51 & - & - & - \\
\hline married & 96 & 54.55 & 80 & 45.55 & 0.90 & 1.04 & $\begin{array}{l}2.03- \\
0.53\end{array}$ \\
\hline \multicolumn{8}{|l|}{ Having a child } \\
\hline yes & 80 & 60.02 & 49 & 39.98 & - & - & - \\
\hline
\end{tabular}




\begin{tabular}{|c|c|c|c|c|c|c|c|}
\hline \multirow{2}{*}{$\begin{array}{l}\text { variables } \\
\text { no }\end{array}$} & \multicolumn{4}{|c|}{ anxiety status } & \multirow{2}{*}{$\begin{array}{l}\mathbf{P} \\
0.005\end{array}$} & \multirow{2}{*}{$\begin{array}{l}\text { OR } \\
0.45\end{array}$} & \multirow{2}{*}{$\begin{array}{l}95 \% \mathrm{Cl} \\
0.78- \\
0.26\end{array}$} \\
\hline & 37 & 42.53 & 50 & 57.47 & & & \\
\hline \multicolumn{8}{|l|}{ Employment status } \\
\hline yes & 90 & 60.40 & 59 & 39.60 & - & - & - \\
\hline no & 29 & 41.43 & 41 & 58.57 & 0.009 & 0.46 & $\begin{array}{l}0.86- \\
0.26\end{array}$ \\
\hline \multicolumn{8}{|l|}{ Overtime(h) } \\
\hline$<50$ & 21 & 55.26 & 17 & 44.74 & - & - & - \\
\hline $50-100$ & 63 & 55.75 & 50 & 44.25 & 0.95 & 1.02 & $\begin{array}{l}2.13- \\
0.48\end{array}$ \\
\hline $100-150$ & 31 & 53.45 & 27 & 46.55 & 0.86 & 0.92 & $\begin{array}{l}2.11- \\
0.40\end{array}$ \\
\hline$>150$ & 4 & 40.00 & 6 & 60 & 0.34 & 0.53 & $\begin{array}{l}2.22- \\
0.13\end{array}$ \\
\hline \multicolumn{8}{|l|}{ hospital } \\
\hline Rouhani Hospital & 77 & 56.20 & 60 & 43.80 & - & - & - \\
\hline Yahya Nejad Hospital & 42 & 51.22 & 40 & 48.78 & 0.47 & 0.81 & $\begin{array}{l}1.41- \\
0.47\end{array}$ \\
\hline \multicolumn{8}{|l|}{ Job satisfaction } \\
\hline yes & 68 & 46.90 & 77 & 53.10 & - & - & - \\
\hline no & 51 & 72.86 & 19 & 27.14 & 0.000 & 3.03 & $\begin{array}{l}5.64- \\
1.63\end{array}$ \\
\hline \multicolumn{8}{|l|}{ Physical activity level } \\
\hline inactive & 16 & 80.00 & 4 & 20 & - & - & - \\
\hline active & 102 & 51.52 & 96 & 48.48 & 0.02 & 0.26 & $\begin{array}{l}0.82- \\
0.08\end{array}$ \\
\hline \multicolumn{8}{|l|}{ exercise } \\
\hline yes & 45 & 43.69 & 58 & 56.31 & & & \\
\hline no & 74 & 63.79 & 42 & 36.21 & 0.003 & 2.27 & $\begin{array}{l}3.90- \\
1.31\end{array}$ \\
\hline \multicolumn{8}{|l|}{ Chronic disease } \\
\hline yes & 17 & 60.71 & 11 & 39.29 & & & \\
\hline
\end{tabular}




\begin{tabular}{|c|c|c|c|c|c|c|c|}
\hline \multirow{2}{*}{$\begin{array}{l}\text { variables } \\
\text { no }\end{array}$} & \multicolumn{4}{|c|}{ anxiety status } & \multirow{2}{*}{$\begin{array}{l}\mathbf{P} \\
0.46\end{array}$} & \multirow{2}{*}{$\begin{array}{l}\text { OR } \\
0.74\end{array}$} & \multirow{2}{*}{$\begin{array}{l}95 \% \mathrm{Cl} \\
1.66- \\
0.32\end{array}$} \\
\hline & 102 & 53.40 & 89 & 46.60 & & & \\
\hline \multicolumn{8}{|c|}{ Workplace violence } \\
\hline yes & 37 & 77.08 & 11 & 22.92 & - & - & - \\
\hline no & 81 & 47.65 & 89 & 52.35 & 0.001 & 0.27 & $\begin{array}{l}0.56- \\
0.12\end{array}$ \\
\hline \multicolumn{8}{|c|}{$\begin{array}{l}\text { Maintain 7-8 h sleep 3-4 times a } \\
\text { week }\end{array}$} \\
\hline yes & 79 & 52.67 & 71 & 47.33 & - & - & - \\
\hline no & 40 & 58.82 & 28 & 41.18 & 0.39 & 1.28 & $\begin{array}{l}2.29- \\
0.71\end{array}$ \\
\hline \multicolumn{8}{|c|}{ Psychiatric disorder } \\
\hline yes & 4 & 80.00 & 1 & 20.00 & - & - & - \\
\hline no & 115 & 53.74 & 99 & 46.26 & 0.27 & 0.29 & $\begin{array}{l}2.64- \\
0.31\end{array}$ \\
\hline \multicolumn{8}{|c|}{ Family history of depression } \\
\hline yes & 13 & 59.09 & 9 & 40.91 & - & - & - \\
\hline no & 105 & 53.27 & 91 & 46.43 & 0.62 & 0.79 & $\begin{array}{l}1.95- \\
0.32\end{array}$ \\
\hline
\end{tabular}

\section{Factors Associated with Depression}

According the correlations between the research variables and the level of depression measured by the binary logistic regression, the variables including age, work experience, job satisfaction, exercising, having chronic diseases, workplace violence and sleep pattern were significantly associated with level of depression. In this regard, the nurses who were older than 35 years had 2.07 times greater risk of getting depression than the nurses who were younger than 35 years; the nurses who had more than 10 years of experience had 1.79 times greater risk of experiencing depression than the nurses who had less than 10 years of experience; and the nurses who were not satisfied with their jobs were 1.7 times as likely to get depression than the nurses who were satisfied with their jobs. Exercising was one of the factors that had a significant relationship with depression. The nurses who did not exercise had 1.76 times greater risk of getting depression than those who exercised. In addition, the nurses who suffered from a chronic disease were at an increased risk for experiencing depression ( $\mathrm{OR}=0.35)$. Moreover, workplace violence and sleep were among the factors associated with depression in nurses who provide care for COVID-19 patients. The nurses who had no violence in their workplace were less likely to get depression than the nurses who experienced workplace violence $(O R=0.39)$ and the nurses who got 7 hours of sleep less than 3 to 4 days a week were more likely to get depression $(O R=1.77)($ Table 6$)$. 
Table 6

Frequency distribution of respondents by depression status and socio-demographic characteristics and other selected variables

\begin{tabular}{|c|c|c|c|c|c|c|c|}
\hline \multirow[t]{3}{*}{ variables } & \multicolumn{4}{|c|}{ depression status } & \multirow[t]{3}{*}{$P$} & \multirow[t]{3}{*}{ OR } & \multirow[t]{3}{*}{$95 \% \mathrm{Cl}$} \\
\hline & yes & $\%$ & no & $\%$ & & & \\
\hline & (n) & & (n) & & & & \\
\hline \multicolumn{8}{|l|}{ sex } \\
\hline male & 25 & 45.45 & 30 & 54.55 & - & - & - \\
\hline female & 68 & 41.21 & 97 & 58.79 & 0.58 & 0.84 & $1.55-0.45$ \\
\hline \multicolumn{8}{|l|}{ age } \\
\hline$\leq 35$ & 54 & 36.49 & 94 & 63.51 & - & - & - \\
\hline$>35$ & 37 & 54.41 & 31 & 45.59 & 0.014 & 2.07 & $3.72-1.15$ \\
\hline \multicolumn{8}{|l|}{ Shift type } \\
\hline morning & 10 & 43.48 & 13 & 56.52 & - & - & - \\
\hline Evening or night & 9 & 42.86 & 12 & 57.14 & 0.96 & 0.97 & $3.21-0.29$ \\
\hline rotational & 74 & 42.29 & 101 & 57.71 & 0.91 & 0.95 & $2.29-0.39$ \\
\hline \multicolumn{8}{|l|}{ Work Experience } \\
\hline$\leq 10$ & 46 & 36.22 & 81 & 63.78 & - & - & - \\
\hline$>10$ & 47 & 50.47 & 46 & 49.46 & 0.03 & 1.79 & $3.10-1.04$ \\
\hline \multicolumn{8}{|l|}{ Education level } \\
\hline bachelor of Nursing & 83 & 44.15 & 105 & 55.85 & - & - & - \\
\hline Master of Nursing & 10 & 31.25 & 22 & 68.75 & 0.17 & 0.57 & $1.28-0.25$ \\
\hline \multicolumn{8}{|l|}{ Marital status } \\
\hline single & 14 & 32.56 & 29 & 67.44 & - & - & - \\
\hline married & 79 & 44.63 & 98 & 55.37 & 0.15 & 1.66 & $3.37-0.82$ \\
\hline \multicolumn{8}{|l|}{ Having a child } \\
\hline yes & 62 & 47.69 & 68 & 52.31 & - & - & - \\
\hline no & 31 & 35.23 & 57 & 64.77 & 0.06 & 0.59 & $1.04-0.34$ \\
\hline \multicolumn{8}{|l|}{ Employment status } \\
\hline yes & 66 & 44.00 & 84 & 56.00 & - & - & - \\
\hline
\end{tabular}




\begin{tabular}{|c|c|c|c|c|c|c|c|}
\hline \multirow{2}{*}{$\begin{array}{l}\text { variables } \\
\text { no }\end{array}$} & \multicolumn{4}{|c|}{ depression status } & \multirow{2}{*}{$\begin{array}{l}P \\
0.44\end{array}$} & \multirow{2}{*}{$\begin{array}{l}\text { OR } \\
0.79\end{array}$} & \multirow{2}{*}{$\begin{array}{l}95 \% \mathrm{Cl} \\
1.42-0.44\end{array}$} \\
\hline & 27 & 38.57 & 43 & 61.43 & & & \\
\hline \multicolumn{8}{|l|}{ Overtime(h) } \\
\hline$<50$ & 13 & 34.21 & 25 & 65.79 & - & - & - \\
\hline $50-100$ & 54 & 48.21 & 58 & 51.79 & 0.13 & 1.79 & $3.85-0.83$ \\
\hline $100-150$ & 20 & 33.33 & 40 & 66.67 & 0.92 & 0.96 & $2.26-0.40$ \\
\hline$>150$ & 6 & 60 & 4 & 40 & 0.14 & 2.88 & $\begin{array}{l}12.07- \\
0.68\end{array}$ \\
\hline \multicolumn{8}{|l|}{ hospital } \\
\hline Rouhani Hospital & 55 & 39.86 & 83 & 60.14 & - & - & - \\
\hline Yahya Nejad Hospital & 38 & 46.34 & 44 & 53.66 & 0.34 & 1.30 & $2.26-0.75$ \\
\hline \multicolumn{8}{|l|}{ Job satisfaction } \\
\hline yes & 56 & 38.36 & 90 & 61.64 & - & - & - \\
\hline no & 36 & 51.43 & 34 & 48.55 & 0.07 & 1.70 & $3.02-0.95$ \\
\hline \multicolumn{8}{|l|}{ Physical activity level } \\
\hline inactive & 8 & 40.00 & 12 & 60 & - & - & - \\
\hline active & 84 & 42.21 & 115 & 57.79 & 0.84 & 1.09 & $2.79-0.42$ \\
\hline \multicolumn{8}{|l|}{ exercise } \\
\hline yes & 36 & 34.95 & 67 & 65.05 & - & - & - \\
\hline no & 57 & 48.72 & 60 & 51.28 & 0.04 & 1.76 & $3.04-1.02$ \\
\hline \multicolumn{8}{|l|}{ Chronic disease } \\
\hline yes & 18 & 64.29 & 10 & 35.71 & - & - & - \\
\hline no & 75 & 39.06 & 117 & 60.94 & 0.014 & 0.35 & $0.81-0.15$ \\
\hline \multicolumn{8}{|l|}{ Workplace violence } \\
\hline yes & 29 & 60.42 & 19 & 39.58 & - & - & - \\
\hline no & 64 & 37.43 & 107 & 62.57 & 0.005 & 0.39 & $0.75-0.20$ \\
\hline \multicolumn{8}{|c|}{$\begin{array}{l}\text { Maintain 7-8 h sleep 3-4 times a } \\
\text { week }\end{array}$} \\
\hline yes & 57 & 38.00 & 93 & 62.00 & - & - & - \\
\hline no & 36 & 52.17 & 33 & 47.83 & 0.050 & 1.77 & $3.16-1.00$ \\
\hline
\end{tabular}




\begin{tabular}{|lccccccc|}
\hline variables & depression status & & P & OR & 95\% Cl \\
\hline Psychiatric disorder & & & & & & & \\
\hline yes & 3 & 60 & 2 & 40 & - & - & - \\
\hline no & 90 & 41.86 & 125 & 58.14 & 0.42 & 0.48 & $2.93-0.07$ \\
\hline Family history of depression & & & & & & & \\
yes & 13 & 59.09 & 9 & 40.91 & - & - & - \\
\hline no & 79 & 40.10 & 118 & 59.90 & 0.09 & 0.46 & $1.13-0.18$ \\
\hline
\end{tabular}

\section{Multivariate Analyses}

In the final model and in the presence of all variables, five variables including marital status, work experience, having children, job satisfaction and history of depression emerged as the significant stressrelated factors. Among these factors, work experience was the strongest variable related to stress in nurses involved with COVID-19, in a way that the nurses who had more than 10 years of experience had a 4 times greater risk of experiencing stress than those nurses who had less than 10 years of experience $(\mathrm{OR}=4.5)$.

Moreover, in the final model and in the presence of all variables, three variables including work experience, job satisfaction and violence in the workplace emerged as the factors associated with anxiety in nurses involved with COVID-19. Among these factors, again work experience had the strongest relationship with anxiety in nurses, in a way that the nurses who had more than 10 years of experience were 3.87 times as likely to experience anxiety as the nurses who had less than 10 years of experience $(\mathrm{AOR}=3.87,95 \% \mathrm{Cl}=$ 2.09-7.18).

Finally, regarding the factors associated with depression, six variables including gender, history of depression, exercising, sleep pattern, history of chronic diseases and violence in the workplace emerged in the final model and in the presence of all variables. The nurses who did not have a history of depression had 0.27 times greater risk of depression than the nurses with a history of depression and the nurses who did not exercise were 2.04 times as likely to suffer from depression as the nurses who did exercise. Moreover, the nurses who got 7 to 8 hours of sleep less than 3 to 4 days a week were 2.28 times as likely to get depressed as the nurses who had a normal sleep pattern and the nurses who did not experience violence in their workplaces and had no history of chronic diseases had respectively 0.39 and 0.32 times greater risk of depression than the nurses who experienced workplace violence and had chronic diseases (Table 7). 
Table 7

Multiple logistic regression model predicting stress, anxiety and depression in nurses involved with Covid-

\begin{tabular}{|c|c|c|c|c|c|}
\hline variables & groups & SE & P_value & AOR & $\begin{array}{l}\text { 95\% Conf. } \\
\text { Interval }\end{array}$ \\
\hline \multicolumn{6}{|l|}{ stress } \\
\hline constant & & 20.27 & 0.37 & 8.42 & $942.42-0.07$ \\
\hline \multirow[t]{2}{*}{ Marital status } & single & - & - & - & - \\
\hline & married & 0.15 & 0.03 & 0.21 & $0.90-0.04$ \\
\hline \multirow[t]{2}{*}{ Years of employment } & $<10$ & - & - & - & - \\
\hline & $\geq 11$ & 1.98 & 0.001 & 4.50 & $10.69-1.89$ \\
\hline \multirow[t]{2}{*}{ Having a child } & yes & - & - & - & - \\
\hline & no & 0.11 & 0.009 & 0.16 & $0.63-0.04$ \\
\hline \multirow[t]{2}{*}{ Job satisfaction } & yes & - & - & - & - \\
\hline & no & 1.08 & 0.01 & 2.66 & $5.90-1.19$ \\
\hline \multirow[t]{2}{*}{ Depression history } & yes & - & - & - & - \\
\hline & no & 0.17 & 0.003 & 0.31 & $0.93-0.10$ \\
\hline \multicolumn{6}{|l|}{ Anxiety } \\
\hline constant & & 0.42 & 0.39 & 0.42 & $3.07-0.05$ \\
\hline \multirow[t]{2}{*}{ work experience } & $<10$ & - & - & - & - \\
\hline & $\geq 11$ & 1.22 & 0.000 & 3.87 & $7.18-2.09$ \\
\hline \multirow[t]{2}{*}{ Job satisfaction } & yes & - & - & - & - \\
\hline & no & 0.92 & 0.004 & 2.67 & $5.25-1.36$ \\
\hline \multirow[t]{2}{*}{ Workplace violence } & yes & - & - & - & - \\
\hline & no & 0.12 & 0.003 & 0.30 & $0.67-0.13$ \\
\hline \multicolumn{6}{|l|}{ Depression } \\
\hline constant & - & 193.08 & 0.005 & 114.02 & $3151.12-4.1$ \\
\hline \multirow[t]{2}{*}{ sex } & male & - & - & - & - \\
\hline & female & 0.20 & 0.10 & 0.55 & $1.14-0.26$ \\
\hline \multirow[t]{2}{*}{ Depression history } & yes & - & - & - & - \\
\hline & no & 0.13 & 0.01 & 0.27 & $0.73-0.10$ \\
\hline
\end{tabular}




\begin{tabular}{|llllll|}
\hline variables & groups & SE & P_value & AOR & $\begin{array}{l}\text { 95\% Conf. } \\
\text { Interval }\end{array}$ \\
\hline exercise & yes & - & - & - & - \\
\cline { 2 - 6 } & no & 0.65 & 0.02 & 2.04 & $3.83-1.08$ \\
\hline $\begin{array}{l}\text { Maintain 7-8 h sleep 3-4 times a } \\
\text { week }\end{array}$ & yes & - & - & - & - \\
\cline { 2 - 6 } & no & 0.73 & 0.01 & 2.28 & $4.27-1.22$ \\
\hline Chronic disease & yes & - & - & - & - \\
\hline Workplace violenc & no & 0.14 & 0.01 & 0.32 & $0.79-0.13$ \\
& yes & - & - & - & - \\
\hline & no & 0.13 & 0.009 & 0.39 & $0.78-0.19$ \\
\hline
\end{tabular}

\section{Discussion}

The results showed that $17.4 \%$ of the nurses had stress, more than half ( $54 \%)$ experienced anxiety and about $43 \%$ had some degrees of depression. In the study of Lai et al. (2020) on the healthcare workers who were involved in providing care for COVID-19 patients in China, $50.4 \%$ of them had symptoms of depression, $44.6 \%$ anxiety, $34 \%$ insomnia, and $71.5 \%$ distress (4). The study of Gupta et al. (2020) in Nepal indicated that $38 \%$ of the healthcare workers involved in caring for COVID-19 patients suffered from anxiety or depression (18). A meta-analysis showed that the prevalence of anxiety and depression in healthcare workers who provide care for COVID-19 patients was $23.2 \%$ and $22.8 \%$ respectively (19). The studies conducted in other parts of the world indicate that the prevalence of anxiety is about 11.3 to $50 \%$ $(21,4,20)$. The findings of the present study report that it is slightly higher than this range. The high prevalence of anxiety in Iranian nurses could be attributed to the lack of protective equipment and fear of infection. Previous studies have also confirmed the high levels of anxiety in those who had direct clinical contact with COVID-19 patients $(18,21)$.

In the present study, prevalence of depression was $43 \%$, which is much higher than what was reported in previous studies in other parts of the world. For instance, the prevalence of depression among healthcare workers was reported to be $8 \%$ in a study in Nepal (18) and $28 \%$ in a study in China (21). Moreover, in a meta-analysis, the combined prevalence was reported to be $22.8 \%$ (19). The high prevalence of depression in Iranian nurses is probably related to risk factors such as the high transmission risk of COVID-19 and the social isolation of nurses due to the fear of transmitting the disease to their family members and friends.

In this study, $17.4 \%$ of the nurses experienced some degrees of stress, which is consistent with the results of the study of Wu et al. (22). However, in the study of Mo et al. (2020) the mean stress score of Chinese nurses was reported to be $39.9 \%$. The long work hours during a week as well as fear of getting infected by 
COVID-19 infection through the respiratory droplets and direct contact (3) increased the Chinese nurses' stress to a relatively higher level compared to Iranian nurses.

Generally, stress in health care workers fighting COVID-19 is attributed to long shifts, unrealistic wages, lack of personal protective equipment, and fear of getting infected or infecting one's family $(23,24)$.

Work experience was the strongest variable associated with stress and anxiety among nurses who were involved with COVID-19 in a way that nurses who had more than 10 years of experience were at an increased risk of experiencing stress and anxiety compared to the nurses with less than 10 years of experience. Long work hours during a week is one of the factors affecting nurses' stress and anxiety (3). The Iranian nurses who were satisfied with their jobs reported less stress, anxiety and depression than those who were not interested in and satisfied with their jobs. In the study of Letavak et al. (2012) it was perceived that the nurses who have a lower level of job satisfaction get more depressed (25). There are other studies that show job satisfaction and professional commitment help to reduce stress and anxiety $(26,27)$. Moreover, a history of depression emerged as a factor associated with stress and depression. Many of the previous studies also showed that high stress levels in nurses lead to anxiety, frustration, depression and other mental and emotional disorders $(28,29)$.

Marital status and having a child in the family were among factors affecting the nurses' stress. Having children causes stress in nurses because the children need care and also because there is a risk of transmission of COVID-19 from the nurse parent to the child, which is in line with the study of Mo (3). Moreover, there was a significant relationship between marital status and nurses' stress, which is consistent with the study of Yildirim et al. (2020) (30).

The nurses who are older than 35 years are more likely to get stress and anxiety. Although in the study of Yildirim et al. (2020), it was shown that the young healthcare staff had higher anxiety scores compared to older ones (30). In addition, the nurses who are informally employed are less likely to experience stress and anxiety than the nurses who are formally employed. The type of employment is associated with workplace anxiety (31). It seems that informally employed nurses can quit their jobs more easily because they have no obligation to the organization. As a result, they have less stress and anxiety than formally employed nurses.

Violence in the workplace emerged as one of the factors associated with stress and anxiety in nurses involved with COVID-19. In the study of Tong et al. (2019), it was found that workplace violence was followed by symptoms of depression in nurses. Reducing the workplace violence and developing the psychological capital can be helpful in the fight against the symptoms of depression (32).

The nurses who exercise and have higher levels of physical activities are less likely to get depression than the nurses who do not exercise and have low levels of physical activities. Physical inactivity and sedentary behavior are significantly associated symptoms of depression and anxiety (33). 
The risk of depression is higher in nurses who have a chronic disease or an abnormal sleep pattern. The study of Levatak et al. (2012) indicates that the nurses who have health problems are more likely to be depressed. In other words, there is a relationship between nurses' medical problems and their depression (25). The study of Wu showed that more than one third of the medical staff had symptoms of insomnia during the COVID-19 outbreak, which was associated to isolation and the psychological distress caused by the prevalence of the disease. Considering various psychological factors, it is necessary for the medical personnel to receive interventions in this regard (34). The distress caused by symptoms of anxiety affects the medical staff's sleep quality (35).

The nurses who fight against COVID-19 are generally under psychological pressure. The authorities should consider the nurses' individual characteristics (age, work experience, gender, marital status, having children and history of chronic diseases), improve their working conditions and provide opportunity for them to get enough sleep to help them modulate their stress, anxiety and depression. Moreover, the nurses should be taught appropriate sports and physical activities to do in their leisure time as well as relaxation techniques to maintain their mental health.

\section{Conclusion}

Prevalence of stress, anxiety and depression is high among nurses involved with caring for COVID-19 patients and it is associated with a number of their individual characteristics including age, work experience, gender, marital status, having children, job satisfaction, workplace violence, sleep, exercising and history of chronic diseases. Therefore, screening services and psychological supports should be provided for nurses and their working conditions should be improved to reduce their anxiety and distress.

\section{Limitations}

The present study has several limitations that should be noted. First, this study only investigated the nurses in the city of Babol in North of Iran. Therefore, the results cannot be generalized to all Iranian nurses. Second, since this was a cross-sectional study, it was not possible to observe the subjects in a longer period of time like a longitudinal study and it could only assess stress, anxiety, and depression at the time of the study. Third, only one questionnaire was used in this study because of the time constraints.

\section{Abbreviations}

COVID-19: Coronavirus disease of 2019; DASS-21: Depression, Anxiety and Stress Scale - 21 items ; MERS: Middle East respiratory syndrome; H1N1: Influenza A virus subtype H1N1; OR: odds ratio; AOR: Adjusted odds ratio; $\mathrm{Cl}$ : Confidence interval

\section{Declarations}




\section{Ethics approval and consent to participate}

The study protocol was approved by the Ethics Committee of the Ethics Committees of Babol University of Medical Sciences (IR.MUBABOL.REC.1399.138) and written informed consent was obtained from the study participants.

\section{Consent for publication}

The article does not contain any individual's details and consent for publication is not applicable.

\section{Availability of data and materials}

All data will be available upon request and consent the Research Deputy of Babol University of Medical Sciences.

\section{Competing interests}

The authors declare that they have no competing interests.

\section{Funding}

This research received a grant from Babol University of Medical Sciences (Grant number: 9909619). The Babol University of Medical Sciences did not have any role in the design of the study and collection, analysis, and interpretation of data and in writing the manuscript.

\section{Authors' contributions}

PAR Study conception/design, carried out the analysis, interpretation of the data and drafting the manuscript. MQQ performed the data analysis and drafting the manuscript. FSZ contributed the data collection. All authors read and approved the final manuscript.

\section{Acknowledgements}

The authors would like to thank the Research Deputy of Babol University of Medical Sciences, Nursing managers and nurses of Ayatollah Rouhani and Shahid Yahya Nejad Hospitals who helped us in this research.

\section{References}


1. Pan, Y., Guan, H., Zhou, S., Wang, Y., Li, Q., Zhu, T., ... Xia, L. Initial CT findings and temporal changes in patients with the novel coronavirus pneumonia (2019-nCoV): A study of 63 patients in Wuhan, China European Radiology.2020; https: //doi.org/10.1007s 00330-020-06731-x.

2. Catton, H., RN, MA, BS (Econ) (Hons). (2020). Global challenges in health and health care for nurses and midwives everywhere. International Nursing Review, 2020; 67(1): 4-6.

3. Mo Y, Deng L, Zhang L, Lang Q, Liao C, Wang N, Qin M, Huang H. Work stress among Chinese nurses to support Wuhan in fighting against COVID-19 epidemic. J Nurs Manag. 2020; 28(5) : 1002-1009. https://doi.org/10.1111/jonm.13014

4. Lai J, Ma S, Wang Y, Cai Z, Hu J, Wei N, Wu J \& etal .Factors Associated With Mental Health Outcomes Among Health Care Workers Exposed to Coronavirus Disease 2019. JAMA Network Open. 2020; 3(3):e203976. https://doi.org/10. 1001/jamanetworkopen.2020.3976.

5. Liu Q, Luo D, Haase JE, Guo Q, Wang X Q, Liu S, Xia L, Liu Z \& etal. The experiences of health-care providers during the COVID-19 crisis in China: a qualitative study. www. thelancet. com/lancetgh Vol 8 June 2020.

6. Chou, L. P., Li, C. Y., \& Hu, S. C. Job stress and burnout in hospital employees: Comparisons of different medical professions, in a regional hospital in Taiwan. British Medical Journal Open.2014: 4,e004185.

7. Khamisa, N., Oldenburg, B., Peltzer, K., Ilic, D. Work related stress, burnout, job satisfaction and general health of nurses International Journal of Environmental Research and Public Health.2015; 12(1): 652-666. https://doi.org/10.3390/ijerp h1201 00652.

8. Kang, L., Li, Y. I., Shaohua, H. U., Chen, M., Yang, C., Yang, B. X., ...Liu ,Z. The mental health of medical workers in Wuhan, China dealing with the 2019 novel coronavirus. Lancet Psychiatry. 2020; 7: e14.

9. Kim Y. Nurses' experiences of care for patients with Middle East respiratory syndrome coronavirus in South Korea. Am J Infect Control. 2018; 46:781-787.

10. Khalid I, Khalid TJ, Qabajah MR, Barnard AG, Qushmaq IA. Healthcare worker semotions, perceived stressors and coping strategies during MERS-CoV outbreak. Clin Med Res. 2016; 1303:1-22.

11. Honey M, Wang WY. New Zealand nurses perceptions of caring for patients with influenza A (H1N1). Nurs Crit Care. 2013;18: 63-69.

12. Spoorthya MS, Pratapa S K, Mahant S. Mental health problems faced by healthcare workers due to the COVID-19 pandemic-A review. Asian Journal of Psychiatry. 51 (2020) 102119. https://doi.org/10.1016/j.ajp.2020.102119

13. Sun N, Wei L, Shi S, Jiao D, Song R,\& etal .A qualitative study on the psychological experience of caregivers of COVID-19 patients. American Journal of Infection Control. 2020; 48 : 592-598.

14. Lovibond PF, Lovibond SH. The structure of negative emotional states comparison of the depression anxiety stress scales (Dass) with the beck depression and anxiety inventories. Behav Res Ther. 1995; 33:335-43. 
15. Mehdipour-Rabori R, Nematollahi M. The effect of recommended Azkar on anxiety, stress, and depression in families of patients undergoing open heart surgery. Iran J Nurs Midwifery Res. 2014;19(3):238-41. pmid: 24949060

16. Najafı Kalyani M, Pourjam E, Jamshidi N, Karimi S, Kalyani VN. Survey of stress, anxiety, depression and self-concept of students of Fasa University of medical sciences, 2010. J Fasa Univ Med Sci. 2013; 3(3):235-40.

17. Shamsaei F, Yaghmaei S, Sadeghian E, Tapak L. Survey of Stress, Anxiety and Depression in Undergraduate Nursing Students of Hamadan University of Medical Sciences. Iranian Journal of Psychiatric Nursing (IJPN). 2018; 6(3):26-30. DOI: 10.21859/ijpn-06034

18. Gupta A K, Mehra A, Niraula A, Kafle K, Deo S P, Singh B, Sahoo S, Grover S. Prevalence of anxiety and depression among the healthcare workers in Nepal during the COVID-19 pandemic. Asian Journal of Psychiatry. $2020 ; 54$ : 102260. doi: 10.1016/j.ajp.2020.102260

19. Pappa S, Ntella V, Giannakas T, Giannakoulis V.G, Papoutsi E, Katsaounou P. Prevalence of depression, anxiety, and insomnia among health care workers during the COVID-19 pandemic: a systematic review and meta-analysis. Brain Behav Immun. 2020.https://doi.org/10.1016/j.bbi. 2020.05.026. S0889-1591(20)30845-X. Advance online publication

20. Zhu J., Sun L., Zhang L., Wang H., FanA., Yang B., Li W., Xiao S. Prevalence and influencing factors of anxiety and depression symptoms in the first-line medical staff fighting against COVID-19 in Gansu. Front. Psychiatry.202; 11, 386. https://doi.org 10.3389/fpsyt.2020.00386

21. Liu, C.Y., Yang, Y.Z., Zhang, X.M., Xu, X., Dou, Q.L., Zhang, W.W., Cheng, A., 2020. The prevalence and influencing factors in anxiety in medical workers fighting COVID-19 in China: a cross-sectional survey. Epidemiol. Infect. 148, e98. https://doi.org/10. 1017/S0950268820001107.

22. Wu, J., Wu, X., Wu, F., Dia, Y., Dechun, C. \& Gong, X. Survey of sleep quality of clinic-al front-line nurses and its influencing factors, in the fight against new coronavirus pneumonia. Nursing Research. 2020; 344, 558- 562.

23. Grover, S., Sahoo, S., Mehra, A., Avasthi, A., Subramanyan, A., 2020. Psychological impact of COVID19 lockdown: an online survey from India. Indian J. Psychiatry

24. Zhang, W.R., Wang, K., Yin, L., Zhao, W.F., Xue, Q., Peng, M., Min, B.Q., Tian, Q., Leng, H.X., Du, J.L., Chang, H., Yang, Y., Li, W., Shangguan, F.F., Yan, T.Y., Dong, H.Q, Han, Y., Wang, Y.P., Cosci, F., Wang, H.X., 2020. Mental health and psychosocial problems of medical health workers during the COVID-19 epidemic in China. Psychother. Psychosom. 1-9. https://doi.org/10.1159/000507639. Advance online publication.

25. Letvak S, Ruhm CJ, Mccoy T. Depression in hospital- employed nurses. Clin Nurse Spec. 2012; 26(3):177-82. doi: 10.1097/NUR.0b013e3182503ef0.

26. Hegney DG, Craigie M, Hemsworth D, Osseiran Moisson R, Aoun S, Francis K, et al. Compassion satisfaction, compassion fatigue, anxiety, depression and stress in registered nurses in Australia: study 1 results. J Nurs Manag. 2014; 22 (4):506-18. 
27. Khodadadi E, Hosseinzadeh M, Roghaye Azimzadeh R, Fooladi M. The relation of depression, anxiety and stress with personal characteristics of nurses in hospitals of Tabriz, Iran. International Journal of Medical Research \& Health Sciences. 2016; 5( 5):140-148.

28. Malinauskiene, V., Leisyte, P., Romualdas, M., \& Kirtiklyte, K. Associations between self-rated health and psychosocial conditions Life style factors and health resources among hospital nurses in Lithuania. Journal of Advanced Nursing. 2011; 67(11), 2383-2393.https:// doi.org/10.1111/j.13652648.2011.05685.x

29. Teles, M. A. B., Barbosa, M. R., Vargas, A. M. D., Gomes, V. E., Ferreira, E. F., Martins, A. M. E.B., ... Ferreira, R. C. Psychosocial work conditions and quality of life among primary health care employees. A cross sectional study. Health Quality of Life Outcomes. 2014: 12, 72. https://doi.org/10.1186/1477-7525-12-72

30. Yildirim TT, Atas O, Asafov A, Yildirim K, Balibey H. J Coll. Physicians Surg Pak. 2020; 30(6):26-31.

31. Choi E S, Jung H S, Kim S H, Park H . The influence of workplace violence on work-related anxiety and depression experience among Korean employees. J Korean Acad Nurs. 2010; 40(5):650-61. doi: 10.4040/jkan.2010.40.5.650.

32. Tong C, Cui C, Li Y,Wang L. The effect of workplace violence on depressive symptoms and the mediating role of psychological capital in chinese township general practitioners and nurses: A cross-sectional study. Psychiatry Investig. 2019 Dec; 16(12): 896-903.

33. Bélair M A, Kohen D E, Kingsbury M, Colman I. Relationship between leisure time physical activity, sedentary behavior and symptoms of depression and anxiety: evidence from a population based sample of Canadian adolescents. BMJ Open. 2018; 8:e021119. doi:10.1136/bmjopen-2017-021119

34. Wu J, Wu X, Wu F, Dia Y, Dechun C,ong X. Survey of sleep quality of clinic-al front-line nurses and its influencing factors, in the fight against new coronavirus pneumonia. Nursing Research. 2020; 344, 558-562. Retrieved from http://kns.cnki.net/kcms/detai I/14.1272.r.20200 214.1136.004.html

35. Kirwan M, Pickett SM, Jarrett NL. Emotion regulation as a moderator between anxiety symptoms and insomnia symptom severity. Psychiatry Res. 2017; 254: 40-7. doi: 10.1016/j.psychres.2017.04.028. 